\title{
Shape and Texture Based Classification of Fish Species
}

\author{
Rasmus Larsen, Hildur Olafsdottir, and Bjarne Kjær Ersbøll \\ DTU Informatics, Technical University of Denmark, \\ Richard Petersens Plads, Building 321, DK-2800 Kgs. Lyngby, Denmark \\ $\{r l$, ho, be $\}$ @imm.dtu.dk
}

\begin{abstract}
In this paper we conduct a case study of fish species classification based on shape and texture. We consider three fish species: cod, haddock, and whiting. We derive shape and texture features from an appearance model of a set of training data. The fish in the training images were manual outlined, and a few features including the eye and backbone contour were also annotated. From these annotations an optimal MDL curve correspondence and a subsequent image registration were derived. We have analyzed a series of shape and texture and combined shape and texture modes of variation for their ability to discriminate between the fish types, as well as conducted a preliminary classification. In a linear discrimant analysis based on the two best combined modes of variation we obtain a resubstitution rate of $76 \%$.
\end{abstract}

\section{Introduction}

In connection with fishery, fishery biological research, and fishery independent stock assessment there is a need for automated methods for determination of fish species in various types of sampling systems. One technique to base such determination on is the use of automated image analysis and classification.

In conjunction with a technology project involving three departments at the Technical University of Denmark: the Departments of Informatics and Mathematical Modelling, Aquatic Systems, and Electrical Engineering, an effort is underway on researching and developing such systems.

Fish phenotype as defined by shape and color-texture both give information on fish species. Systematic description of differences in fish morphology dates back to the seminal work by d'Arcy Thompson [1]. Glasbey [2 demonstrate how a registration framework can be used to discriminate been the fish species whiting and haddock.

Modelling and automated registration of classes of biological objects with respect to shape and texture is elegantly achieved by the active appearance models 3] (AAM). The training of AAMs is based on sets of images with the objects of interests marked up by a series of corresponding landmarks. Developments of the original algorithms have aimed at alleviating the cumbersome work involved in manually annotating the training set. One such efforts is the 
minimum description length (MDL) approach to finding coordinate correspondences between surves and surfaces proposed by Davies et al 4. A variant of this approach including curvature information was proposed by Thodberg [5].

\section{Data}

The study described in this article is based on a sample of 108 fish: 20 cod (torsk), 58 haddock (kuller), and 30 whiting (hviling) caugth in Kattegat. The fish were imaged using a standard color CCD camera under a standardized white light illumination. Example images are shown in Fig. 1. All fish images were mirrored to face left before further analysis.

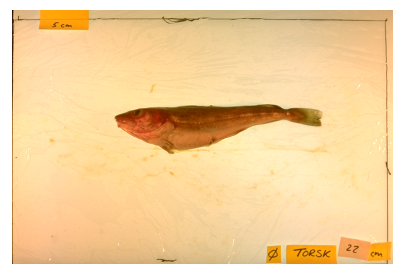

(a) Cod, in Danish torsk

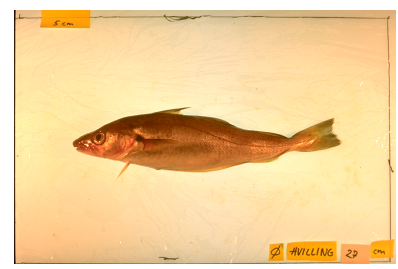

(b) Whiting, in Danish hvilling

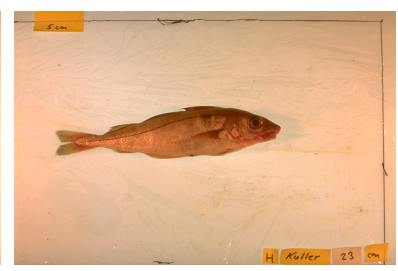

(c) Haddock, in Danish kuller

Fig. 1. Example images of the three types of fish considered in the article. Note the differences in the shape of the snout as well as the abscence of the thin dark line in the cod that is present in haddock and whiting.

\section{Methods and Results}

The fish images were contoured with the red and green curves shown in Fig. 2 Additionally, the fish eye centre was marked (the blue landmark). The two curves from the training set were input to the MDL based correspondence analysis by Thodberg [5], and the resulting landmarks recorded. Note that the landmarks are placed such that we have equi-distant sampling along the curves on the mean shape. This landmark annotated mean fish was then subjected to a Delaunay triangulation [6] and piece-wise affine warps of the corresponding triangles on each fish shape to the resulting Delaunay triangles of the mean shape constitute the training set registration. The quality of this registration is illustrated in Fig. 3. In this image each pixel is the log-transformed variance of each color

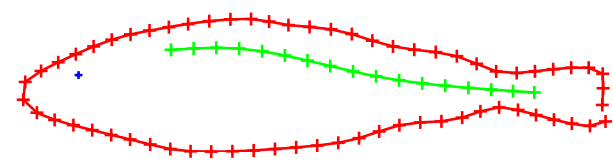

Fig. 2. The mean fish shape. The landmarks are placed according to a MDL principle. 


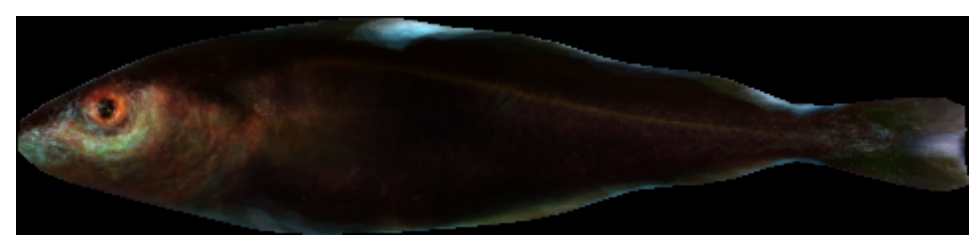

Fig. 3. Model variance in each pixel explaining the texture variability in the training set after registration

across the training set after this registration. As can be seen the texture variation is concentrated in the fish head along the spine, and at fins.

Following this step an AAM was trained. The resulting first modes of variation are shown in Figs. 4 (shape alone), 5 (texture only), and 6 (combined shape and texture variation). The combined principal component analysis weigh the shape and texture according to the generalized variances of the two types of variation. Note, for the shape as well as for the combined model that the first factor captures a mode of variation pertaining to a bending of the fish body, i.e. a variation not related to fish specie. The second combined factor primarily captures the fish snout shape variation, and the third mode the presence/abscence of the black line along the fish body.

We next subject the principal component scores to a pairwise Fisher discriminant analysis [7] in order to evaluate the potential in discriminating between these species based on image analysis. The Fisher discriminant score explain the ability of a particular variable to discriminate between a particular pair of classes. From Table 1 we wee that it is overall most difficult to discriminate between Haddock-Whiting, texture is better for discriminating between Haddock-Cod, and combined shape and texture better for Cod-Whiting.

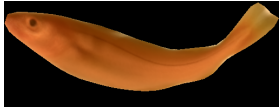

(a)

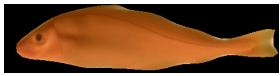

(d)

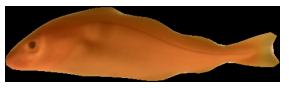

(g)

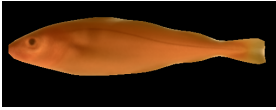

(b)

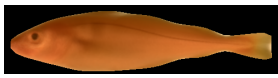

(e)

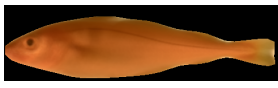

(h)

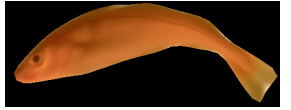

(c)

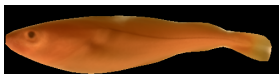

(f)

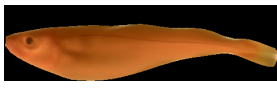

(i)

Fig. 4. First three shape modes of variance. (b,e,h) mean shape; (a,d,g) -3 standard deviations; (c,f,i) +3tandard deviations. 


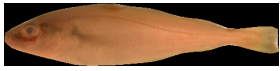

(a)

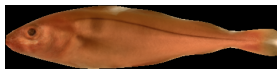

(d)

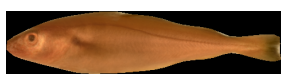

(g)

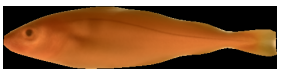

(b)

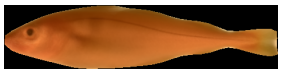

(e)

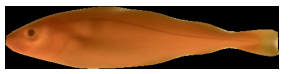

(h)

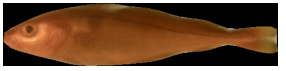

(c)

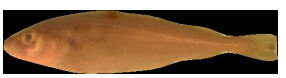

(f)

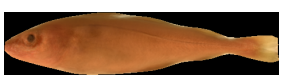

(i)

Fig. 5. First three texture modes of variance. (b,e,h) mean shape; (a,d,g) -3 standard deviations; $(\mathrm{c}, \mathrm{f}, \mathrm{i})+3$ tandard deviations.

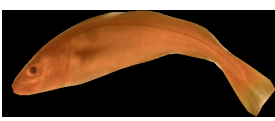

(a)

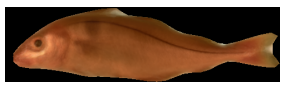

(d)

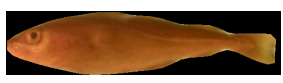

$(\mathrm{g})$

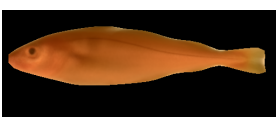

(b)

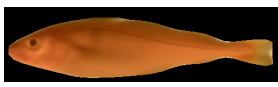

(e)

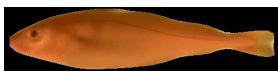

(h)

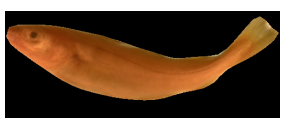

(c)

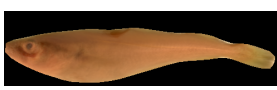

(f)

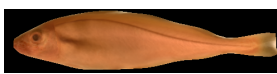

(i)

Fig. 6. First three combined shape and texture modes of variance. (b,e,h) mean shape; $(\mathrm{a}, \mathrm{d}, \mathrm{g})-3$ standard deviations; $(\mathrm{c}, \mathrm{f}, \mathrm{i})+3$ tandard deviations.

Table 1. Best univariate Fisher scores for each pair of classes

\begin{tabular}{l|ccc} 
& Haddock-Whiting Haddock-Cod Cod-Whiting \\
\hline Texture & $1.4303(\mathrm{pc} 2)$ & $5.0709(\mathrm{pc} 2)$ & $4.9675(\mathrm{pc} 3)$ \\
Shape & $1.2905(\mathrm{pc} 3)$ & $1.7616(\mathrm{pc} 2)$ & $1.3085(\mathrm{pc} 4)$ \\
Combined & $1.3536(\mathrm{pc} 2)$ & $2.6492(\mathrm{pc} 3)$ & $5.7519(\mathrm{pc} 3)$
\end{tabular}

Finally, the best two factors from the combined shape and texture model were applied in a linear discriminant analysis. The resubstitution matrix of the classification is shown in Table 2, and the classification result is illustrated in Fig. 7. The overall resubstitution rate is $76 \%$. The major confusion is between haddock and whiting. These numbers are of course somewhat optimistic given that no test on an independent test set is carried out. On the other hand the amount of parameter tuning to the training set is kept at a minimum. 
Table 2. Resubstitution matrix for a linear discriminant analysis

\begin{tabular}{|c|c|c|c|}
\hline & \multicolumn{3}{|c|}{ Cod Haddock Whiting } \\
\hline Cod & 18 & 2 & 0 \\
\hline Haddock & 2 & 40 & 16 \\
\hline Whiting & 0 & 6 & 24 \\
\hline
\end{tabular}

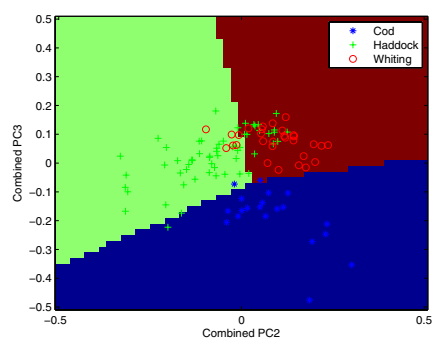

Fig. 7. Classification result for a linear discriminant analysis

\section{Conclusion}

In this paper we have provided an initial account of a procedure for fish species classification. We have demonstrated that to some degree shape and texture based classification can be use to discriminate between the fish species cod, haddock, and whiting.

\section{References}

1. Thompson, D.W.: On Growth and Form, 2nd edn. (1942) (1st edn. 1917)

2. Glasbey, C.A., Mardia, K.V.: A penalized likelihood approach to image warping. Journal of the Royal Statistical Society, Series B 63, 465-514 (2001)

3. Cootes, T.F., Edwards, G.J., Taylor, C.J.: Active appearance models. IEEE T. on Pattern Analysis and Machine Intelligence 23(6), 681-685 (2001)

4. Davies, R.H., Twining, C.J., Cootes, T.F., Waterton, J., Taylor, C.J.: A minimum description length approach to statistical shape modelling. IEEE Transactions on Medical Imaging (2002)

5. Thodberg, H.H.: Minimum description length shape and appearance models. In: Proc. Conf. Information Processing in Medical Imaging, pp. 51-62. SPIE (2003)

6. Delaunay, B.: Sur la sphère vide. Otdelenie Matematicheskikh i Estestvennykh Nauk, vol. 7, pp. 793-800 (1934)

7. Fisher, R.A.: The use of multiple measurements in taxonomic problems. Annals of Eugenics 7, 179-188 (1936) 